\title{
US health care utilization and costs in adult patients with atopic dermatitis by disease severity
}

\author{
Xin Wang, PhD; Natalie N Boytsov, PhD; Magdaliz Gorritz, MPH; William N Malatestinic, PharmD; \\ Orin M Goldblum, MD; and Rolin L Wade, MS
}

\section{What is already known about this subject}

- Atopic dermatitis (AD) is associated with significant patient burden related to direct medical costs.

- Given the trend towards increasing AD prevalence, updated estimates are needed, especially among AD patients with different disease severity.

\section{ABSTRACT}

BACKGROUND: Although previous studies have reported the economic burden of atopic dermatitis (AD) in adults, updates are needed using more current data and measure of disease severity.

OBJECTIVE: To describe the health care resource utilization (HCRU) and associated costs in US adults diagnosed with AD overall and by disease severity.

METHODS: This real-world retrospective study identified adults aged at least 18 years who received a clinical diagnosis of $A D$ in a dermatology electronic medical record

\section{What this study adds}

- Annual all-cause total direct health care costs for US adults diagnosed with $A D$ were $\$ 10,474$ per patient per year from 2016 to 2018.

- Patients with severe disease had significantly higher all-cause annual health care costs $(\$ 23,242)$ than patients with clear to mild disease $(\$ 8,936)$.

- This study provided health care costs estimates by severity in AD patients, which could help inform cost-effective treatment decisions.

\section{Author affiliations}

Xin Wang, PhD; Magdaliz Gorritz, MPH; and Rolin L Wade, MS, IQVIA, Inc., Plymouth Meeting, PA. Natalie N Boytsov, PhD; William N Malatestinic, PharmD; and Orin M Goldblum, MD, Eli Lilly and Company, Indianapolis, IN.

AUTHOR CORRESPONDENCE: Magdaliz Gorritz, 267.640.6291

magdaliz.gorritz@iqvia.com

J Manag Care Spec Pharm 2022;28(1):69-77

Copyright @2022, Academy of Managed Care Pharmacy. All rights reserved.

(EMR) database between 2016 and 2018 (first record=index date), which was linked to an administrative claims database. Patients were required to have an $A D$ diagnostic code and at least 6 months of continuous enrollment in medical and pharmacy benefits before and after the index date. Baseline severity was assessed using the Physician Global Assessment score closest to the index date. Inpatient and outpatient services, visits to specialists and its seasonality, treatment use, and associated annual direct health care costs were reported using descriptive statistics.

RESULTS: Annual all-cause direct health care costs were $\$ 10,474$ per patient per year and primarily driven by outpatient visits and pharmacy use. Compared with patients with clear to mild disease, more AD patients with severe disease had at least 1 dermatology $(73.0 \%$ vs $58.5 \%$ ) and allergy/immunology office visit ( $16.0 \%$ vs $5.5 \%$ ) and AD-related medications ( $90.0 \%$ vs $64.3 \%$ ). All-cause total annual costs in patients with severe disease $(\$ 23,242)$ were significantly higher than in patients with clear to mild disease $(\$ 8,936 ; P=0.0002)$. Little seasonal variation in dermatology office visits was observed.

CONCLUSIONS: Significant economic burden primarily driven by outpatient and pharmacy utilization was observed in AD patients, which increased with disease severity. 
Atopic dermatitis (AD) is a chronic pruritic inflammatory skin condition substantially affecting quality of life (QoL). ${ }^{1}$ $\mathrm{AD}$ has been considered to be a pediatric disease, since roughly $80 \%$ of patients have disease onset by age 6 years. ${ }^{2}$ However, recent evidence shows that $\mathrm{AD}$ is prevalent in adults, affecting an estimated $7 \%$ of US adults. ${ }^{3}$ Patients diagnosed with $\mathrm{AD}$ have decreased health-related QoL and an increased risk of several co-occurring conditions compared with the general population. ${ }^{3,4}$ Asthma and allergic rhinitis are common comorbidities of $\mathrm{AD} .^{3}$

The economic impact of $\mathrm{AD}$ is difficult to measure because of the broad disease severity levels and multiple cost contributors related to indirect and direct costs. Drucker et al assessed health care resource utilization (HCRU) and costs associated with AD in US adults and found that $\mathrm{AD}$ was associated with adjusted total incremental annual costs of $\$ 3,302 .{ }^{5} \mathrm{HCRU}$ and costs were higher in the more severe group, with adjusted total incremental annual costs of $\$ 4,463$. Using 2013 US National Health and Wellness Survey data, another study reported that AD patients had significantly greater HCRU and total costs compared with non-AD patients. ${ }^{6}$

Substantial gaps still remain in understanding the effect that $\mathrm{AD}$ has on the lives of adults,,${ }^{7,8}$ particularly by disease severity. Although previous studies assessed the economic burden of AD, current data are lacking. Drucker et al examined health care costs by disease severity, in which severity was measured based on AD treatments. ${ }^{5}$ Previous studies have proxied $\mathrm{AD}$ severity based on past treatment, specifically defining more severe AD as the use of phototherapy or systemic immunomodulatory medications. However, a valid algorithm to define AD severity is still lacking. $5,7,8$

The purpose of this study was to describe the HCRU and associated costs overall and by disease severity between 2016 and 2018 among US adult patients diagnosed with $\mathrm{AD}$, using electronic medical records (EMR) linked to an administrative claims database.

\section{Methods}

\section{DATA SOURCES}

Data were derived from Modernizing Medicine's Electronic Medical Assistant (EMA), a specialty-specific EMR database for dermatology, and the IQVIA PharMetrics Plus $(\mathrm{P}+)$ claims database. EMA encompasses over 42 million unique patients with at least 1 visit across the United States and contains structured data from over 9,000 dermatology providers. P+ contains adjudicated medical and pharmacy claims for over 150 million health plan members across the United States from 2006 onwards and is representative of the US commercially insured population.

All patient-level data were deidentified by Modernizing Medicine Data Services, Inc. (MMDS) and IQVIA in accordance with the Health Insurance Portability and Accountability Act of 1996 (HIPAA). Data from EMA were used to identify patients with $\mathrm{AD}$, establish disease severity, and assess seasonality of dermatology office visits. The $\mathrm{P}+$ database was used to assess HCRU, including concomitant medication use, and health care costs during follow-up.

Deterministic matching was used to link EMA to the $\mathrm{P}+$ database using actual patient information to ensure continuity of patient records across datasets. In alignment with privacy standards to ensure that patients could not be identified by linking 2 data sources, all dates were shifted by plus or minus 15 days in each data source. This shifting introduced up to a 30-day discrepancy between visits observed in EMA and P+. In addition, another 15 days were added to the index identification window in $\mathrm{P}+$ to allow for additional time associated with claims submission.

\section{STUDY POPULATION}

The study population consisted of adults aged at least 18 years with a clinical AD diagnosis (International Classification of Diseases, Tenth Revision, Clinical Modification [ICD-10-CM] codes L20.0, L20.8, L20.89, and L20.9) in EMA between January 1, 2016, and June 30, 2018 (first diagnosis date was designated as the index date), who were linked to the $\mathrm{P}+$ database. Patients were required to have at least 6 months of pre- and post-index continuous enrollment in $\mathrm{P}+$ and at least 1 claim with an $\mathrm{AD}$ diagnosis code within 45 days of index, accounting for index date shifting after linking EMA data to $\mathrm{P}+$.

\section{BASELINE AD SEVERITY}

Severity of AD was defined by the Physicians' Global Assessment (PGA) score reported in EMA. PGA is a physician-reported measure that assesses overall disease severity at a given time point on a 5-point scale that ranges from 0 to 4 , where 0 indicates clear, 1 indicates almost clear, 2 indicates mild, 3 indicates moderate, and 4 indicates severe AD. ${ }^{9}$ Baseline severity was defined as the last PGA score before or on the index date. Patients were grouped into 3 categories based on baseline severity: clear to mild (0-2), moderate (3), and severe (4).

\section{HCRU AND COSTS}

All-cause HCRU was measured during available follow-up and was reported as per patient per year (PPPY), stratified by baseline severity. All-cause HCRU included inpatient visits, total hospital days, outpatient visits stratified by 


\section{FIGURE 1 Patient Attrition}

Exclude patients aged $<2$ years, $n=54,568$

Exclude patients with data quality issues in $E M A, n=116,468$
Exclude patients with $<6$ months of continuous enrollment in PharMetrics Plus before and after index date, $n=147,816$

Exclude patients with data quality issues in PharMetrics Plus, $n=200$

Exclude patients with no claims in PharMetrics Plus within 45 days of index date with a diagnosis of $A D, n=29,422$

Exclude patients aged $<18$ years, $n=2,984$
Patients with $\geq 1$ record in the MMDS database with an

ICD-10-CM diagnosis code of L20.x that is not a rule-out

diagnosis between January 1, 2016, and June 30, 2018

(first diagnosis date is index date) $\mathrm{N}=1,261,449 ; 100.0 \%$

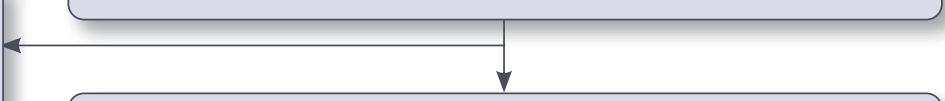

Patients aged $>2$ years with no data quality issues in EMA $\mathrm{n}=1,090,413 ; 86.4 \%$

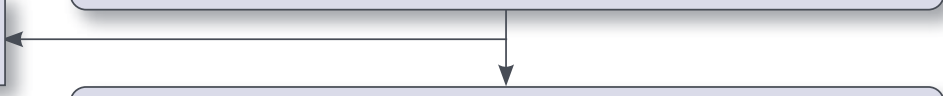

Patients with linkage to PharMetrics Plus $\mathrm{n}=185,206 ; 14.7 \%$

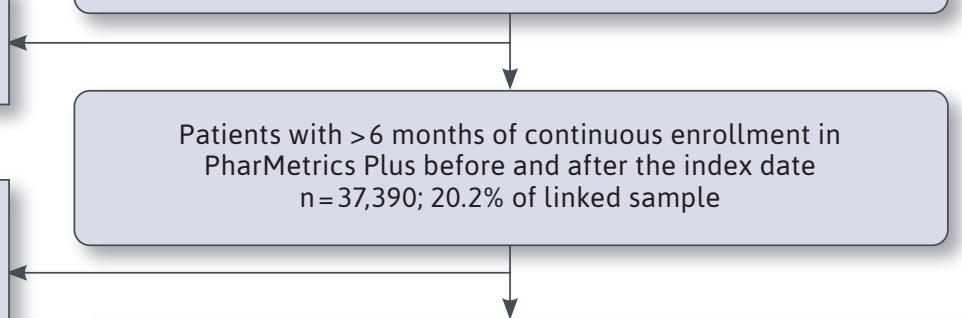

Patients with a clinical diagnosis of AD in EMA and a claim with a diagnosis of $A D$ in PharMetrics Plus within 45 days of the index date $\mathrm{n}=7,768 ; 4.2 \%$ of linked sample

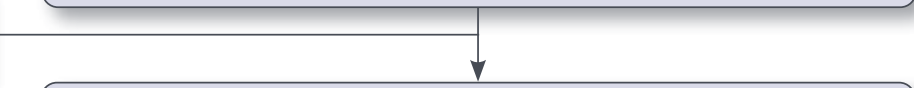

Final study population $n=4,784 ; 2.6 \%$ of linked sample dermatology and allergy/immunology visits, phototherapy administration, emergency department (ED) visits, pharmacy utilization overall, and AD-related treatments (Supplementary Table 1, available in online article). Use of concomitant medications was assessed during the pre- and post-index periods. Medications assessed included asthma medications, antihypertensives, antidiabetics, antidepressants, anti-anxiety medications, antibiotics, antivirals, antifungals, sleep aids, and pain medications.

Seasonality of dermatology office visits observed in EMA was assessed over the entire study period. Office visits occurring on the same date were counted as 1 unique visit.
Visits were grouped into seasons by date, according to the meteorological definition of spring as March-May, summer as June-August, autumn as September-November, and winter as December-February.

All-cause total direct health care costs were assessed during the follow-up period overall and by resource categories (inpatient, outpatient, ED, phototherapy administration, and pharmacy) and were reported as PPPY. Costs were reported in 2019 US dollars based on allowed amounts of adjudicated claims, including the amount paid by the plan plus out-of-pocket costs. 


\section{STATISTICAL ANALYSIS}

This study was descriptive in nature. Mean, SD, and median were reported for continuous variables. Frequency and percentages were reported for categorical variables. Student's t-test and chi-square tests were used to compare continuous and categorical variables, respectively, between patients with clear to mild vs moderate disease and clear to mild vs severe disease. The Benjamini-Hochberg procedure was used to calculate the adjusted $P$ values by adjusting the false discovery rate. All analyses were performed using SAS version 9.4 (SAS Institute).

\section{Results}

\section{STUDY POPULATION}

A total of 1,261,449 patients with at least 1 record in the MMDS database with an ICD-10-CM code of L20.x were identified, of which 1,090,413 were aged at least 2 years. Among them, 185,206 (17\%) were linked to the $\mathrm{P}+$ database (Figure 1). Patients linked to $\mathrm{P}+$ were younger (mean $[\mathrm{SD}]=37.9$ [21.5] vs 42.8 [24.3]; $\mathrm{P}<0.0001)$, and a higher proportion was female $(61.9 \%$ vs $60.5 \%$; $P<0.0001)$ than in the unlinked sample (Supplementary Table 2, available in online article).

After applying inclusion and exclusion criteria, the linked cohort consisted of 4,784 adults with $\mathrm{AD}$ diagnosis. The mean (SD) age was 42.3 (15.2), and 64.7\% were female. Patients were primarily from the South (45.9\%) and Midwest (26.0\%; Table 1). Half of the patients were indexed in 2017 (50.4\%). The mean (SD) disease duration was 68.3 (142.9) months (Table 1). The most common comorbidities were allergic contact dermatitis (67.3\%), depression (38.4\%), and allergic rhinitis (36.2\%; Table 1).

A total of 626 (13.1\%) patients had baseline PGA scores available; more than half had moderate $(40.6 \%)$ or severe (16.0\%) disease; and $43.5 \%$ had clear to mild disease. Baseline total body surface area (BSA) was reported for 320 (6.7\%) patients. Among these, $33.1 \%$ had BSA less than $10 \%$; $13.4 \%$ had BSA greater than 50\%; $86.6 \%$ had BSA $50 \%$ or less; and $53.5 \%$ had BSA of $10 \%$ or more and $50 \%$ or less. The most common lesion locations were arms (35.8\%), trunk (27.9\%), and legs (26.2\%; Table 1).

\section{HEALTH CARE RESOURCE UTILIZATION}

Among the 4,784 AD patients, $6.4 \%$ had at least 1 hospitalization with a mean (SD) of 6.6 (13.5) hospital days during the mean follow-up of 470 days. Overall, $98.3 \%$ of patients had a least 1 outpatient visit/service; $65.0 \%$ had at least 1 visit to a dermatologist; and $6.5 \%$ had at least 1 visit to an allergist/immunologist. Nearly $20 \%$ of patients had at least
1 ED visit (19.5\%). Pharmacy utilization was high, with $91.5 \%$ of patients having at least 1 prescription. AD-related therapies accounted for most of the pharmacy utilization-72.9\% had at least 1 claim for $\mathrm{AD}$ treatment, and $67.2 \%$ and $31.4 \%$ had at least 1 prescription claim for AD topical therapy and systemic therapy, respectively (Supplementary Table 3, available in online article).

Differences in HCRU were observed by disease severity (Supplementary Table 3). Among severe patients, 73.0\% had at least 1 dermatology office visit compared with $58.5 \%$ of patients with clear to mild disease $(P=0.034)$. Likewise, $16.0 \%$ of severe patients had at least 1 allergist/immunologist office visit compared with $5.5 \%$ of patients with clear to mild disease $(P=0.0044)$. Severe patients had higher AD medication use than patients with clear to mild disease (90.0\% vs $64.3 \%$; $P=0.0005$ ), including use of topical therapies $(81.0 \%$ vs $57.0 \% ; P=0.0005)$ and systemic therapies (63.0\% vs $25.0 \% ; \mathrm{P}=0.0005)$.

The most common concomitant medications used during baseline were antibiotics (24.7\%), followed by antidepressant or anti-anxiety medications (24.1\%) and antihypertensives (21.9\%). Also, 5.0\% used pain medications and 3.2\% used sleep aides. During follow-up, the most common medications received were antibiotics (43.9\%), followed by antidepressant or anti-anxiety medications (31.8\%) and antihypertensives (26.3\%). In addition, $13.4 \%$ of patients used pain medications, and 5.2\% used sleep aides during follow-up (Supplementary Table 4 , available in online article).

A higher proportion of severe patients used asthma (24.0\% vs $9.6 \% ; \mathrm{P}=0.0003)$ and anti-anxiety medications (32.0\% vs $20.6 \% ; P=0.0217)$ pre-index than patients with clear to mild disease. During follow-up, higher proportions of severe patients used asthma medications $(54.0 \%$ vs $15.8 \% ; \mathrm{P}<0.0001)$ than patients with clear to mild disease. Conversely, a lower proportion of patients with moderate disease used antihypertensives $(18.9 \%$ vs $27.6 \%$; $\mathrm{P}=0.0188)$ and antibiotics $(39.8 \%$ vs $48.5 \%$; $P=0.0431)$ than clear to mild patients. Use of pain medications, sleep aids, and antidepressant medications was not statistically different among severity groups (Supplementary Table 4).

Little seasonal variation was observed in dermatology office visits (Supplementary Figure 1, available in online article). Overall, the highest proportion of visits occurred between September and November (26.0\%) and the lowest proportion of visits occurred between December and February (23.9\%). A similar distribution of visits was observed by US region. 


\section{TABLE 1 Baseline Demographic and Clinical Characteristics}

\begin{tabular}{|c|c|c|c|c|c|c|c|c|c|c|}
\hline \multirow[b]{3}{*}{ Measures } & \multirow{2}{*}{\multicolumn{2}{|c|}{\begin{tabular}{|c|} 
All adult AD patients \\
$\mathrm{N}=\mathbf{4 , 7 8 4}$
\end{tabular}}} & \multirow{2}{*}{\multicolumn{2}{|c|}{$\begin{array}{c}\text { Clear to Mild } \\
n=272\end{array}$}} & \multirow{2}{*}{\multicolumn{2}{|c|}{$\begin{array}{c}\text { Moderate } \\
n=254\end{array}$}} & \multirow{2}{*}{\multicolumn{2}{|c|}{$\begin{array}{l}\text { Severe } \\
n=100\end{array}$}} & \multirow{3}{*}{$\begin{array}{c}P \text { value } \\
\text { (clear to } \\
\text { mild vs } \\
\text { moderate) }\end{array}$} & \multirow{3}{*}{$\begin{array}{l}\text { P value } \\
\text { (clear to } \\
\text { mild vs } \\
\text { severe) }\end{array}$} \\
\hline & & & & & & & & & & \\
\hline & $\mathbf{n}$ & $\%$ & $\mathbf{n}$ & $\%$ & $\mathbf{n}$ & $\%$ & $\mathbf{n}$ & $\%$ & & \\
\hline \multicolumn{11}{|l|}{ Age (years) } \\
\hline Mean & 42.3 & & 39.9 & & 41.3 & & 41.2 & & 0.2909 & 0.4857 \\
\hline SD & 15.2 & & 14.7 & & 14.6 & & 14.7 & & & \\
\hline Median & 44.0 & & 40.0 & & 43.0 & & 39.0 & & 0.2365 & 0.5305 \\
\hline \multicolumn{11}{|l|}{ Age categories (years) } \\
\hline $18-34$ & 1,632 & 34.1 & 110 & 40.4 & 88 & 34.6 & 38 & 38.0 & 0.0196 & 0.9522 \\
\hline $35-44$ & 854 & 17.9 & 62 & 22.8 & 47 & 18.5 & 23 & 23.0 & & \\
\hline $45-54$ & 1,049 & 21.9 & 41 & 15.1 & 67 & 26.4 & 14 & 14.0 & & \\
\hline $55-64$ & 1,034 & 21.6 & 46 & 16.9 & 46 & 18.1 & 21 & 21.0 & & \\
\hline $65-75$ & 164 & 3.4 & $<5$ & - & $<5$ & - & $<5$ & - & & \\
\hline$\geq 75$ & 51 & 1.1 & $<5$ & - & $<5$ & - & $<5$ & - & & \\
\hline \multicolumn{11}{|l|}{ Gender } \\
\hline Female & 3,095 & 64.7 & 183 & 67.3 & 159 & 62.6 & 51 & 51.0 & 0.2606 & 0.0040 \\
\hline Male & 1,689 & 35.3 & 89 & 32.7 & 95 & 37.4 & 49 & 49.0 & & \\
\hline \multicolumn{11}{|l|}{ Geographic region } \\
\hline Northeast & 467 & 9.8 & 28 & 10.3 & 22 & 8.7 & 11 & 11.0 & 0.6070 & 0.1582 \\
\hline Midwest & 1,242 & 26.0 & 82 & 30.1 & 67 & 26.4 & 34 & 34.0 & & \\
\hline South & 2,197 & 45.9 & 127 & 46.7 & 126 & 49.6 & 35 & 35.0 & & \\
\hline West & 878 & 18.4 & 35 & 12.9 & 39 & 15.4 & 20 & 20.0 & & \\
\hline \multicolumn{11}{|l|}{ Payer type } \\
\hline Commercial & 2,813 & 58.8 & 155 & 57.0 & 124 & 48.8 & 60 & 60.0 & 0.1714 & 0.6245 \\
\hline Self-insured & 1,901 & 39.7 & 115 & 42.3 & 128 & 50.4 & 40 & 40.0 & & \\
\hline Medicare risk & 22 & 0.5 & $<5$ & - & $<5$ & - & $<5$ & - & & \\
\hline Medicaid & 47 & 1.0 & $<5$ & - & $<5$ & - & $<5$ & - & & \\
\hline Unknown & $<5$ & - & $<5$ & - & $<5$ & - & $<5$ & - & & \\
\hline \multicolumn{11}{|l|}{ Index year } \\
\hline 2016 & 1,000 & 20.9 & 61 & 22.4 & 45 & 17.7 & 12 & 12.0 & 0.2256 & 0.0803 \\
\hline 2017 & 2,410 & 50.4 & 135 & 49.6 & 123 & 48.4 & 56 & 56.0 & & \\
\hline 2018 & 1,374 & 28.7 & 76 & 27.9 & 86 & 33.9 & 32 & 32.0 & & \\
\hline $\begin{array}{l}\text { Patients with nonmissing } \\
\text { disease duration }\end{array}$ & 162 & 3.39 & 12 & 4.41 & 23 & 9.06 & 12 & 12.0 & 0.0327 & 0.0083 \\
\hline \multicolumn{11}{|l|}{ Disease duration, months } \\
\hline Mean & 68.3 & & 18.1 & & 88.0 & & 89.0 & & 0.0302 & 0.1248 \\
\hline SD & 142.9 & & 32.8 & & 103.6 & & 150.5 & & & \\
\hline
\end{tabular}




\section{TABLE 1 Baseline Demographic and Clinical Characteristics (continued)}

\begin{tabular}{|c|c|c|c|c|c|c|c|c|c|c|}
\hline \multirow[b]{3}{*}{ Measures } & \multirow{2}{*}{\multicolumn{2}{|c|}{\begin{tabular}{|c|} 
All adult AD patients \\
$\mathrm{N}=\mathbf{4 , 7 8 4}$
\end{tabular}}} & \multirow{2}{*}{\multicolumn{2}{|c|}{$\begin{array}{c}\text { Clear to Mild } \\
\qquad n=272\end{array}$}} & \multirow{2}{*}{\multicolumn{2}{|c|}{$\begin{array}{c}\text { Moderate } \\
n=254\end{array}$}} & \multirow{2}{*}{\multicolumn{2}{|c|}{$\begin{array}{l}\text { Severe } \\
n=100\end{array}$}} & \multirow{3}{*}{$\begin{array}{c}\text { P value } \\
\text { (clear to } \\
\text { mild vs } \\
\text { moderate) }\end{array}$} & \multirow{3}{*}{$\begin{array}{l}\text { P value } \\
\text { (clear to } \\
\text { mild vs } \\
\text { severe) }\end{array}$} \\
\hline & & & & & & & & & & \\
\hline & $\mathbf{n}$ & $\%$ & $\mathbf{n}$ & $\%$ & $\mathbf{n}$ & $\%$ & $\mathbf{n}$ & $\%$ & & \\
\hline \multicolumn{11}{|c|}{ Charlson Comorbidity Index score } \\
\hline Mean & 0.4 & & 0.4 & & 0.3 & & 0.4 & & 0.1295 & 0.9812 \\
\hline SD & 0.9 & & 1.0 & & 0.6 & & 1.1 & & & \\
\hline Median & 0.0 & & 0.0 & & 0.0 & & 0.0 & & & \\
\hline $0-1$ & 4,407 & 92.1 & 246 & 90.4 & 242 & 95.3 & 94 & 94.0 & 0.0211 & 0.3013 \\
\hline 2 & 232 & 4.8 & 16 & 5.9 & $<5$ & - & $<5$ & - & & \\
\hline $3+$ & 145 & 3.0 & 10 & 3.7 & $<5$ & - & $<5$ & - & & \\
\hline \multicolumn{11}{|l|}{ Comorbid conditions } \\
\hline Allergic contact dermatitis & 3,219 & 67.3 & 177 & 65.1 & 193 & 76.0 & 89 & 89.0 & 0.0062 & $<0.0001$ \\
\hline Depression & 1,837 & 38.4 & 100 & 36.8 & 87 & 34.3 & 35 & 35.0 & 0.5474 & 0.7537 \\
\hline Allergic rhinitis & 1,730 & 36.2 & 95 & 34.9 & 93 & 36.6 & 46 & 46.0 & 0.6865 & 0.0510 \\
\hline Pruritis & 1,535 & 32.1 & 88 & 32.4 & 100 & 39.4 & 41 & 41.0 & 0.0933 & 0.1203 \\
\hline Asthma & 1,413 & 29.5 & 79 & 29.0 & 81 & 31.9 & 46 & 46.0 & 0.4784 & 0.0021 \\
\hline $\begin{array}{l}\text { Hypertension/high blood } \\
\text { pressure }\end{array}$ & 1,267 & 26.5 & 80 & 29.4 & 58 & 22.8 & 22 & 22.0 & 0.0866 & 0.1554 \\
\hline $\begin{array}{l}\text { Infection of skin and } \\
\text { subcutaneous tissue }\end{array}$ & 1,144 & 23.9 & 58 & 21.3 & 66 & 26.0 & 36 & 36.0 & 0.2082 & 0.0039 \\
\hline Anxiety & 1,131 & 23.6 & 71 & 26.1 & 48 & 18.9 & 23 & 23.0 & 0.0484 & 0.5415 \\
\hline Obesity & 540 & 11.3 & 32 & 11.8 & 26 & 10.2 & 6 & 6.0 & 0.5760 & 0.1036 \\
\hline Baseline PGA score & 626 & 13.1 & 272 & 100.0 & 254 & 100.0 & 100 & 100.0 & & \\
\hline 0 - clear & 34 & 5.4 & 34 & 12.5 & & & & & $<0.0001$ & $<0.0001$ \\
\hline 1 - almost clear & 31 & 5.0 & 31 & 11.4 & & & & & & \\
\hline 2 - mild & 207 & 33.1 & 207 & 76.1 & & & & & & \\
\hline 3 - moderate & 254 & 40.6 & & & 254 & 100.0 & & & & \\
\hline 4 - severe & 100 & 16.0 & & & & & 100 & 100.0 & & \\
\hline Baseline BSA score & 320 & 6.7 & 64 & 23.5 & 118 & 46.5 & 66 & 66.0 & $<0.0001$ & $<0.0001$ \\
\hline Mean & 23.6 & & 6.7 & & 22.6 & & 45.9 & & $<0.0001$ & $<0.0001$ \\
\hline SD & 24.4 & & 6.5 & & 21.4 & & 27.0 & & & \\
\hline Median & 15.0 & & 5.0 & & 15.0 & & 40.0 & & $<0.0001$ & $<0.0001$ \\
\hline $\mathrm{BSA}<10 \%$ & 106.0 & 33.1 & 48 & 75.0 & 30 & 25.4 & $<5$ & - & $<0.0001$ & $<0.0001$ \\
\hline $10 \% \leq \mathrm{BSA}<15 \%$ & 44.0 & 13.8 & 7 & 10.9 & 21 & 17.8 & 6 & 9.1 & 0.2207 & 0.7257 \\
\hline$B S A \geq 15 \%$ & 170.0 & 53.1 & 9 & 14.1 & 67 & 56.8 & 58 & 87.9 & $<0.0001$ & $<0.0001$ \\
\hline $\mathrm{BSA} \leq 50 \%$ & 277 & 86.6 & 64 & 100.0 & 106 & 89.8 & 42 & 63.6 & 0.0083 & $<0.0001$ \\
\hline $\mathrm{BSA}>50 \%$ & 43 & 13.4 & $<5$ & - & 12 & 10.2 & 24 & 36.4 & 0.0083 & $<0.0001$ \\
\hline
\end{tabular}




\section{TABLE 1 Baseline Demographic and Clinical Characteristics (continued)}

\begin{tabular}{|c|c|c|c|c|c|c|c|c|c|c|}
\hline \multirow[b]{3}{*}{ Measures } & \multirow{2}{*}{\multicolumn{2}{|c|}{\begin{tabular}{c|} 
All adult AD patients \\
$\mathrm{N}=\mathbf{4 , 7 8 4}$
\end{tabular}}} & \multirow{2}{*}{\multicolumn{2}{|c|}{$\begin{array}{c}\text { Clear to Mild } \\
n=272\end{array}$}} & \multirow{2}{*}{\multicolumn{2}{|c|}{$\begin{array}{c}\text { Moderate } \\
n=254\end{array}$}} & \multirow{2}{*}{\multicolumn{2}{|c|}{$\begin{array}{l}\text { Severe } \\
n=100\end{array}$}} & \multirow{3}{*}{$\begin{array}{c}\text { P value } \\
\text { (clear to } \\
\text { mild vs } \\
\text { moderate) }\end{array}$} & \multirow{3}{*}{$\begin{array}{l}\text { P value } \\
\text { (clear to } \\
\text { mild vs } \\
\text { severe) }\end{array}$} \\
\hline & & & & & & & & & & \\
\hline & $\mathbf{n}$ & $\%$ & $\mathbf{n}$ & $\%$ & $\mathbf{n}$ & $\%$ & $n$ & $\%$ & & \\
\hline \multicolumn{11}{|l|}{ AD location } \\
\hline $\operatorname{arm}^{\mathrm{a}}$ & 1,713 & 35.8 & 103 & 37.9 & 110 & 43.3 & 43 & 43.0 & 0.2041 & 0.3688 \\
\hline trunk $^{\mathrm{b}}$ & 1,334 & 27.0 & 66 & 24.3 & 106 & 41.7 & 46 & 46.0 & $<0.0001$ & $<0.0001$ \\
\hline legc & 1,253 & 26.2 & 64 & 23.5 & 93 & 36.6 & 33 & 33.0 & 0.0010 & 0.0651 \\
\hline face $^{d}$ & 789 & 16.5 & 50 & 18.4 & 54 & 21.3 & 23 & 23.0 & 0.4077 & 0.3201 \\
\hline hande & 688 & 14.4 & 29 & 10.7 & 61 & 24.0 & 13 & 13.0 & $<0.0001$ & 0.5275 \\
\hline \multicolumn{11}{|c|}{ Treatments dispensed within $\mathbf{4 5}$ days before or any time after index date $\left(P_{+}\right)$} \\
\hline $\begin{array}{l}\text { Patients treated with } \\
\text { topical therapies only }\end{array}$ & 2,131 & 44.5 & 119 & 43.8 & 105 & 41.3 & 24 & 24.0 & 0.5762 & 0.0005 \\
\hline Topical corticosteroids & 2,035 & 42.5 & 110 & 40.4 & 97 & 38.2 & 23 & 23.0 & 0.5973 & 0.0019 \\
\hline $\begin{array}{l}\text { Topical } \\
\text { immunosuppressants }\end{array}$ & 267 & 5.6 & 18 & 6.6 & 15 & 5.9 & 7 & 7.0 & 0.7364 & 0.8961 \\
\hline Topical PDE4 inhibitors & 110 & 2.3 & 8 & 2.9 & 14 & 5.5 & $<5$ & - & 0.1411 & 0.6188 \\
\hline $\begin{array}{l}\text { Patients treated with } \\
\text { systemic therapies with or } \\
\text { without topical therapies }\end{array}$ & 2,029 & 42.4 & 109 & 40.1 & 115 & 45.3 & 70 & 70.0 & 0.2279 & $<0.0001$ \\
\hline Oral immunosuppressants & 165 & 3.4 & 6 & 2.2 & 17 & 6.7 & 20 & 20.0 & 0.0119 & $<0.0001$ \\
\hline $\begin{array}{l}\text { Injectable } \\
\text { immunosuppressants }\end{array}$ & 7 & 0.1 & $<5$ & - & $<5$ & - & $<5$ & - & 0.3003 & \\
\hline Systemic steroids & 1,927 & 40.3 & 105 & 38.6 & 101 & 39.8 & 51 & 51.0 & 0.7852 & 0.0317 \\
\hline Biologic therapy & 173 & 3.6 & $<5$ & - & 28 & 11.0 & 37 & 37.0 & $<0.0001$ & $<0.0001$ \\
\hline Topical corticosteroids & 1,726 & 36.1 & 82 & 30.1 & 92 & 36.2 & 60 & 60.0 & 0.1390 & $<0.0001$ \\
\hline $\begin{array}{l}\text { Topical TCI (note: } \\
\text { same as topical } \\
\text { immunosuppressant) }\end{array}$ & 287 & 6.0 & 9 & 3.3 & 21 & 8.3 & 21 & 21.0 & 0.0143 & $<0.0001$ \\
\hline Topical PDE4 inhibitors & 129 & 2.7 & $<5$ & - & 11 & 4.3 & 8 & 8.0 & 0.0215 & 0.0005 \\
\hline Phototherapy & 73 & 1.5 & $<5$ & - & 11 & 4.3 & 5 & 5.0 & 0.0005 & 0.0002 \\
\hline $\begin{array}{l}\text { Patients with no topical or } \\
\text { systemic therapy within } \\
45 \text { days before or any } \\
\text { time after index } \\
\text { (ie, no treatment) }\end{array}$ & 624 & 13.0 & 44 & 16.2 & 34 & 13.4 & 6 & 6.0 & 0.3682 & 0.0107 \\
\hline
\end{tabular}

Note: Cell counts $<5$ were included in the chi-square analysis. Data shown are given as $n, \%$ unless otherwise noted.

aBody location =arm, shoulder, upper arm.

'Body location = trunk, back, hip, abdomen.

'Body location=leg, thigh.

${ }^{\mathrm{d} B o d y}$ location = face, eyelid, ear, lip, nose.

${ }^{e}$ Body location $=$ hand, finger, fingernail.

$A D=$ atopic dermatitis; $B S A=$ body surface area; $P D E 4=$ phosphodiesterase type 4; $P G A=$ Physicians' Global Assessment; $T C l=$ topical calcineurin inhibitor. 


\section{HEALTH CARE COSTS}

Annual all-cause total direct health care costs were $\$ 10,474$ PPPY, primarily driven by outpatient (\$4,546 PPPY) and pharmacy (\$3,886 PPPY) costs. AD-related medication costs totaled \$1,168 PPPY, of which \$254 and \$914 were attributed to topical and systemic therapies (Supplementary Table 5, available in online article).

Annual all-cause total direct health care costs across the severity subgroups ranged from $\$ 8,936$ for clear to mild patients and $\$ 11,755$ for moderate patients to $\$ 23,242$ for severe patients. A significant difference in total all-cause costs occurred between clear to mild and severe patients $(\mathrm{P}<0.0001)$. ED (\$600 PPPY) and pharmacy (\$16,186 PPPY) costs for severe patients were significantly higher than for clear to mild patients (\$202 PPPY, $P=0.0201$ and $\$ 3,575$ PPPY, $\mathrm{P}=0.0002$, respectively; Supplementary Table 5).

\section{Discussion}

This real-world study provides updated HCRU and associated costs in adults diagnosed with $\mathrm{AD}$, overall and by disease severity, using commercial claims linked to dermatology EMR data. Annual all-cause total direct health care costs were \$10,474 per patient, with higher costs among patients with higher severity (clear to mild: \$8,936; moderate: \$11,755; and severe: $\$ 23,242)$. Health care costs were driven by outpatient visits (\$4,546 PPPY) and pharmacy costs $(\$ 3,885$ PPPY). AD-related medication costs totaled \$1,168 PPPY, of which $\$ 914$ were attributed to systemic therapy. During the follow-up, 31.8\%, $13.4 \%$, and $5.2 \%$ of patients used antidepressant/anti-anxiety medications, pain medications, and sleep aids, respectively. Although little seasonal variation in occurrence of dermatology office visits existed, a slightly higher number of visits occurred in autumn months.

Previous literature has provided cost estimates using older data and proxies for disease severity. Drucker et al used adjudicated claims from 2013 to determine the economic burden of $\mathrm{AD}$ in adults. ${ }^{5}$ Disease severity was approximated based on treatments received during followup rather than on clinical measures. Furthermore, Drucker et al used ICD-9-CM code 691.8 (other atopic dermatitis and related conditions), a broader billing code than the ICD-10-CM codes used in the current study.

Despite these differences, the current study results are consistent with Drucker et al's findings. ${ }^{5}$ Outpatient services and pharmacy had the highest frequency of utilization and annual health care costs, which were similar across the 2 studies when accounting for inflation. Eckert et al used the 2013 National Health and Wellness Survey data to evaluate the burden of AD in adults. ${ }^{6}$ Survey responders who reported having $\mathrm{AD}$ in the previous 12 months diagnosed by a physician were included. Severity was self-graded as mild, moderate, or severe. HCRU was defined by the number of health care provider visits, and direct costs were estimated using 2012 Medical Expenditure Panel Survey data. While Eckert et al did not find a significant difference in burden by severity, findings by severity may have been limited by a small patient sample.

\section{LIMITATIONS}

This study provides HRCU and health care costs for adult AD patients overall and by severity using recent claims data; however, results should be interpreted in the context of its limitations. First, data used in this study were not collected for research purposes, and only a small proportion of patients had available measures of disease severity. These gaps in severity data may limit the generalizability to the entire AD population, particularly if patients with available measures of disease severity had more severe disease manifestations that warranted the clinical assessment. Furthermore, health care providers do not always interpret PGA severity in the same way and may not be trained in its use; therefore, variations may exist in the severity assessment. Also, the MMDS database includes data captured only from dermatologists contributing to the EMR network, so results may not be generalizable to all patients with AD treated by other specialties or primary care physicians.

Similarly, the $\mathrm{P}+$ database has an underrepresentation of patients aged over 65 years and does not include data on uninsured patients, which further reduces the generalizability to Medicare or uninsured patients. In our study, only the patients who could be linked between MMDS and P+ data were assessed for their health care utilization and costs; those who could not be linked were not assessed, so difference may exist between the linked and unlinked patients.

Finally, administrative claims provide data on direct medical costs associated with payments for medical visits and prescriptions but do not capture over-the-counter costs and indirect medical costs such as loss in productivity.

\section{Conclusions}

This study provided updated information on the economic burden of AD in US adults, overall and stratified by disease severity, from 2016 to 2018. The results show that AD costs are largely driven by outpatient use and pharmacy costs and increase with higher severity, consistent with results published in previous studies. Additional studies are needed to further evaluate indirect costs associated with AD. 


\section{DISCLOSURES}

This work was sponsored by Eli Lilly and Company. Gorritz and Wade are employees of IQVIA, which was contracted by Eli Lilly and Company to conduct this study and develop the manuscript. Wang was employed by IQVIA at the time of this study. Malatestinic and Goldblum are employees and stockholders of Eli Lilly and Company. Boytsov was an employee of Eli Lilly at the time of this research.

\section{ACKNOWLEDGMENTS}

The authors thank Wei-Ti Huang and Yiyun Lin for their statistical and programming expertise.

\section{REFERENCES}

1. Bylund S, Von Kobyletzki LB, Svalstedt M, Svensson A. Prevalence and incidence of atopic dermatitis: a systematic review. Acta Derm Venereol. 2020;100(12):adv00160.
2. Weidinger S, Beck LA, Beiber T, et al. Atopic dermatitis. Nat Rev Dis Primers. 2018;4(1):1.

3. Silverberg, JI. Atopic dermatitis in adults. Med Clin North Am. 2020;104(1): 157-76.

4. Eckert L, Gupta S, Amand C, et al. Impact of atopic dermatitis on healthrelated quality of life and productivity in adults in the United States: an analysis using the National Health and Wellness Survey. J Am Acad Dermatol. 2017;77(2):274-79.

5. Drucker AM, Qureshi AA Amand C, et al. Health care resource utilization and costs among adults with atopic dermatitis in the United States: a claims-based analysis. J Allergy Clin Immunol Pract. 2018;6(4):1342-48.

6. Eckert L, Gupta S, Amand C, et al. The burden of atopic dermatitis in US adults: health care resource utilization data from the 2013 National Health and Wellness Survey. J Am Acad Dermatol. 2018;78(1): 54-61.e1. doi:10.1016/j.jaad.2017.08.002
7. Aaron M, Drucker MD, Box GD. Research gaps in quality of life and economic burden of atopic dermatitis: the National Eczema Association Burden of Disease Audit. JAMA Dermatol. 2016;152(8):873-4. doi:10.1001/ jamadermatol.2016.1978

8. Drucker AM, Wang AR, Li WQ, Sevetson E, Block JK, Qureshi AA. The burden of atopic dermatitis: summary of a report for the National Eczema Association. J Invest Dermatol. 2017;137(1):26-30.

9. Gooderham MJ, Bissonnette R, Grewal P, et al. Approach to the assessment and management of adult patients with atopic dermatitis: a consenus document. Section II: tools for assessing the severity of atopic dermatitis. Journal of Cutaneous Medicine and Surgery. 2018;22(1 suppl):10S-16S. 